\title{
Uncommon complications of biliary stones
}

\author{
S. Janssen ${ }^{1}$, I. van Mierlo ${ }^{1}$, L. P. L. Gilissen ${ }^{1}$, S. W. Nienhuijs ${ }^{2}$, J. Heemskerk ${ }^{3}$ \\ ${ }^{1}$ Department of Gastroenterology and Hepatology, Catharina Hospital, Eindhoven, The Netherlands \\ ${ }^{2}$ Department of Surgery, Catharina Hospital, Eindhoven, The Netherlands \\ ${ }^{3}$ Department of Surgery, Laurentius Hospital, Roermond, The Netherlands \\ Email: sj.janssen@chello.nl
}

Received 7 July 2011; revised 16 November 2011; accepted 25 November 2011

\begin{abstract}
Gallstone disease has a high incidence, and most common presentations are well known and recognized. Particulalry in the elderly population though, uncommon presentations of gallstone disease are more frequent, and can easily be missed or misinterpreted. In this article we present 5 such patients with an atypical presentation of gallstone disease. We will then discuss atypical gallstone disease in more detail.
\end{abstract}

Keywords: Gallstone Disease; Gallstone Complications; Elderly

\section{INTRODUCTION}

Gallstones are crystalline formations by accretion or concretion of bile components. The occurrence has been reported up to 25 percent [1]. This may, however, not be a realistic representation of the prevalence, since most gallstones are asymptomatic: only $10 \%-30 \%$ of biliary stones give symptoms [1], leading to aspecific abdominal or dyspeptic complaints, cholecystitis, cholangitis and pancreatitis. Patient history and clinical examination are still pivotal in diagnosing symptomatic gallstone disease. Usually the symptoms are readily recognized, after which cholecystectomy is performed. However, not all gallstone disease comes with a clear-cut presentation, and not all abdominal complaints are attributable to the presence of gallstones. Diagnostic tools such as ultrasound examination, computed tomography scan or magnetic resonance cholangio and pancreaticography may not always reveal gallstone disease [2]. This rings especially true for the elderly patient, in which complicated gallstone disease is more common, and disease presentation or the disease itself may be atypical.

As gallstone disease may result in serious complications, it remains important to recognize even these uncommon presentations. This case-series describes five such patients with infrequent complications of biliary stones, in order to improve awareness.

\section{CASES}

\subsection{Patient A}

A 76-year old woman visited the Emergency Ward of our hospital with abdominal pain, constipation and vomiting since one week. Her medical history revealed two previous admissions for postprandial pain in her upper abdomen, nausea, vomiting and diarrhoea. These episodes had lasted for two weeks and dissolved spontaneously.

Physical examination now revealed a general tenderness of her abdomen, without distention of the abdomen or palpable masses. Laboratory analysis showed leucocytosis and moderately elevated CRP (Table 1). A plain abdominal X-ray revealed a big round calcification in the right upper abdomen, which was moved to the left upper abdomen on a second X-ray (Figure 1). An abdominal CT scan was performed and showed a dilated stomach, as well as a collapsed gallbladder with a

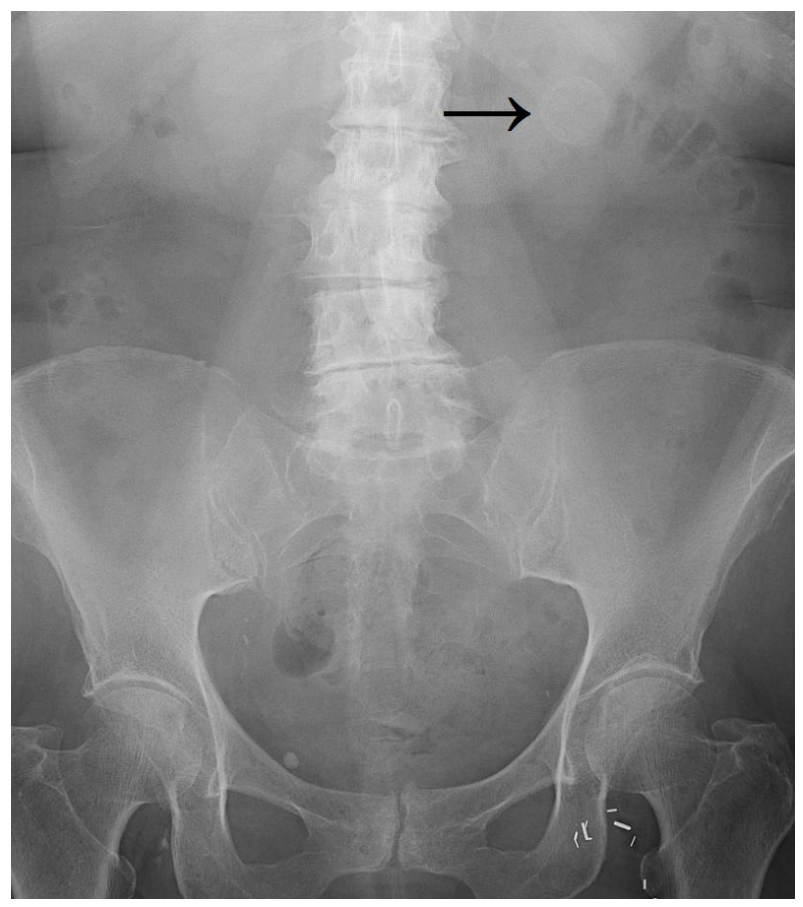

Figure 1. The calcification is visible in the upper left abdomen. 
Table 1. Laboratory results on presentation.

\begin{tabular}{|c|c|c|c|c|c|c|}
\hline & & Patient A & Patient B & Patient C & Patient D & Patient E \\
\hline $\mathrm{Hb}$ & $\mathrm{mmol} / \mathrm{L}$ & 7.7 & 7.8 & 8.9 & 8.9 & 6.5 \\
\hline Leukocytes & $/ \mathrm{nL}$ & 12 & 17 & 11 & 6 & 27 \\
\hline CRP & $\mathrm{mg} / \mathrm{L}$ & 68 & 49 & 100 & 64 & 22 \\
\hline Bilirubin & $\mu \mathrm{mol} / \mathrm{L}$ & 16 & 51 & 14 & 21 & 7 \\
\hline ASAT & $\mathrm{U} / \mathrm{L}$ & 36 & 190 & 26 & 21 & 20 \\
\hline ALAT & $\mathrm{U} / \mathrm{L}$ & 45 & 120 & 33 & 12 & 12 \\
\hline $\mathrm{AF}$ & $\mathrm{U} / \mathrm{L}$ & 61 & 197 & 120 & 43 & 128 \\
\hline$\gamma$-GT & $\mathrm{U} / \mathrm{L}$ & 58 & 170 & 58 & 19 & 42 \\
\hline Amylase (serum) & $\mathrm{U} / \mathrm{L}$ & & & & & 2200 \\
\hline Amylase (urine) & $\mathrm{U} / \mathrm{L}$ & & & & & 4400 \\
\hline
\end{tabular}

calcified mass. In the jejunum an obstructing calcified mass was seen of $2.6 * 2.3 \mathrm{~cm}$ (Figure 2), with distension of the proximal jejunum. These findings established the diagnosis of gallstone ileus. Retrospectively, the stone was already detectable on a previous abdominal $\mathrm{X}$-ray nine months earlier. The patient was diagnosed with an intermittent gallstone ileus. She underwent a laparotomy with enterolithotomy (Figure 3) and recovered fully.

Her gallbladder was not resected due to the surrounding tissues being inflammated at the time of surgery. No further symptoms were seen in a 2-year follow-up.

\subsection{Patient B}

An 88-year old woman presented with malaise. Her medical history included a sliding hernia and diverticulosis. Apart from frequent coughing, there were no other specific complaints. Physical examination showed no abnormalities. Laboratory analysis showed elevated inflammation markers and signs of cholestasis (Table 1). Abdominal ultrasound as well as CT-scan revealed an abscess located in the right liver lobe (Figure 4), with a slender biliary duct, sludge in both the gallbladder and biliary duct. The abscess was drained. Cultures showed an E. coli, for which Amoxicillin/clavulanic acid was administered for 6 weeks. The patient made a complete recovery.

Several months later she was admitted again, this time septic due to cholangitis. An ERCP showed several large bile duct stones with clear dilation (Figure 5). After papillotomy the stones were removed with a basket. A second ERCP was performed because of renewed signs of inflammation and cholestasis. No more ductal stones were found. A regimen of amoxicillin/clavulanic acid was administered for 2 days, after which patient re- covered.

Due to the extensive choledocholithiasis, it was concluded that the previous liver abscess was a complication of gall stone disease, as well. Remarkably, both ultrasound and CT-scan had not revealed any stones or

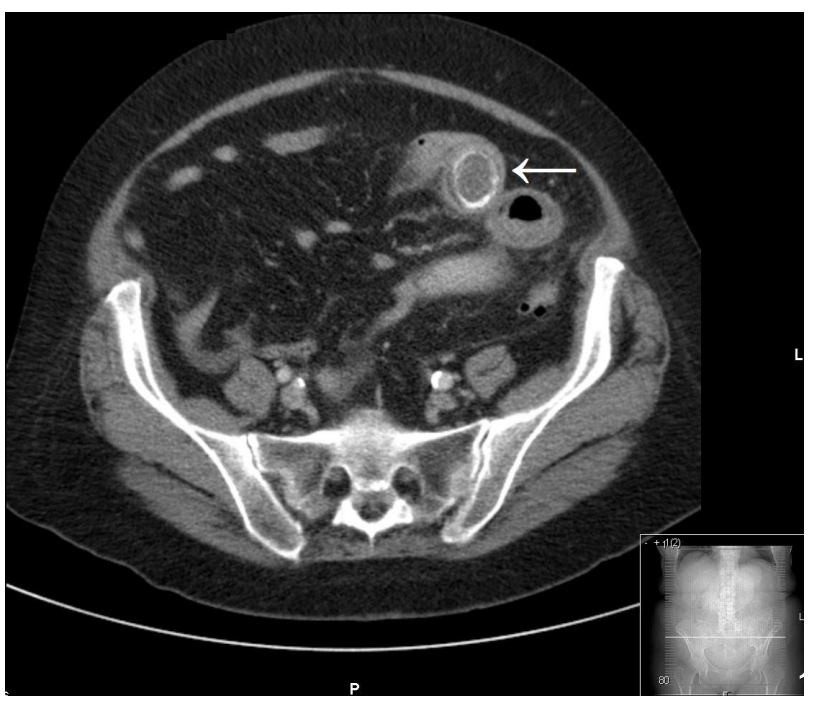

Figure 2. CT scan showing the intestinally located gallstone.

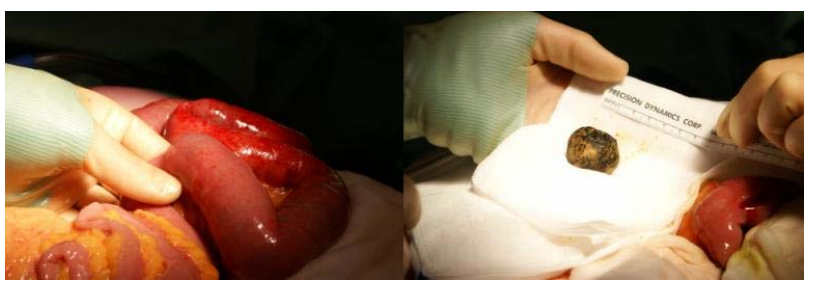

(a)

Figure 3. (a) and (b) preoperative photographs of gallstone ileus (note the prestenotic dilated and congestive loops with intraluminal stone en lean postobstructive bowel loops). 


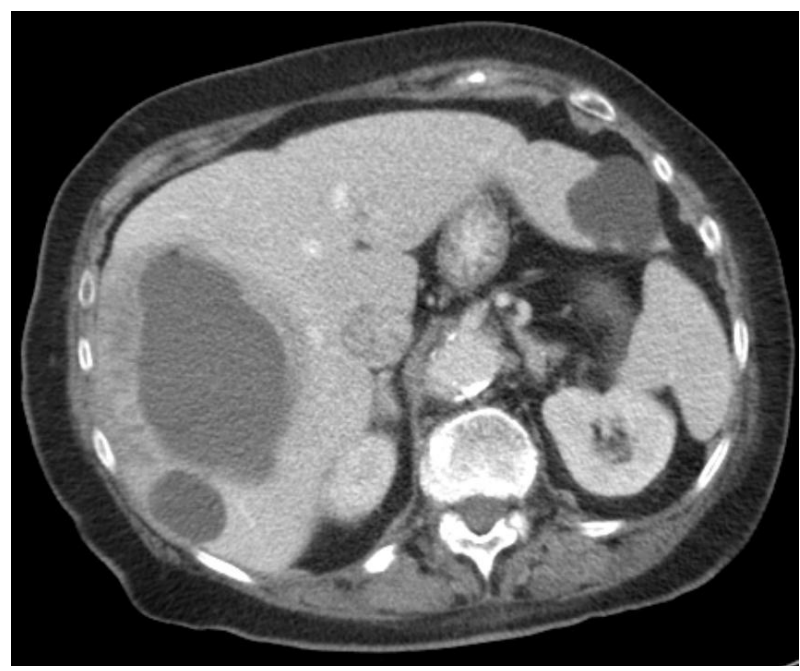

Figure 4. Liver abcess and cysts.

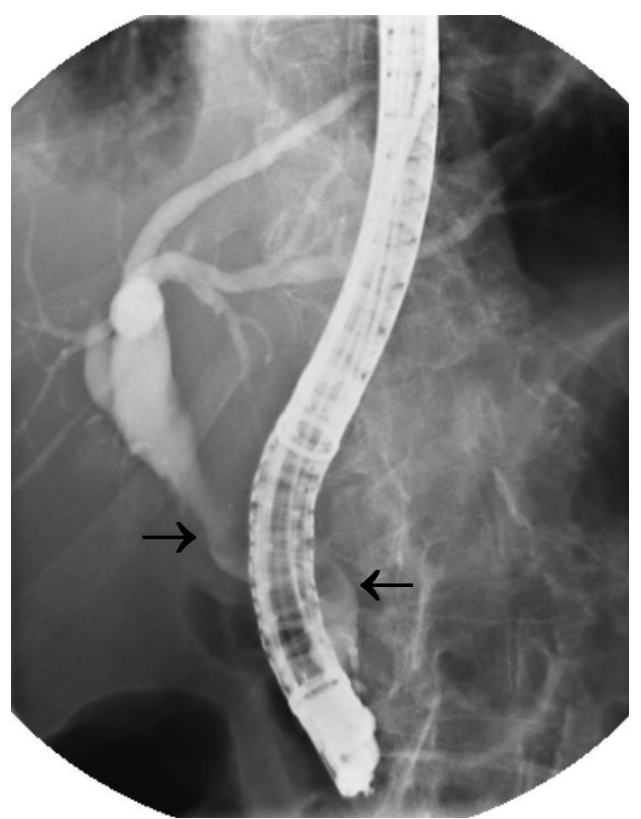

Figure 5. ERCP with multiple biliary duct stones.

bile duct dilation. It is highly unlikely for the patient to have formed multiple new gall stones so soon after the liver abcess.

Cholecystectomy was proposed, but patient refused. No new complications have been seen in a 1 year follow up.

\subsection{Patient C}

An 83-year old male was referred to our hospital for a second opinion. His medical history included an abdominal aortic endoprothesis, atrial fibrillation and laparoscopic cholecystectomy three years earlier. Patient had a weight loss of 10 kilograms, productive coughing, dyspnea, and sometimes vomiting after a severe cough- ing stroke. No fever was reported. Claritromycin administered by the general practitioner for suspected respiratory infection had given no relief. Physical examination showed no abnormalities beyond bilateral basal crackles and a median abdominal herniation without signs of incarceration. Lab results showed mild leucocytosis, elevated CRP and a slightly elevated gamma (Table 1). Chest X-ray showed an infiltration in the right lower quadrant, and an elevated right hemidiaphragm. A scan had already been performed by the referring hospital, which showed a fluid-filled cavity, positioned dorsally in the liver. This was originally thought to be a bilioma. A new CT scan with intravenous contrast showed the cavity to be an abscess with two densities inside. These were thought to be gall stones, spilled during the cholecystectomy. The abscess was percutaneously drained by CT guidance, and in a second stage evacuated by operation. During this procedure the two densities proved to be gall stones (Figure 6). Retrospectively, they had also been visible on an earlier CT scan (Figure 7).

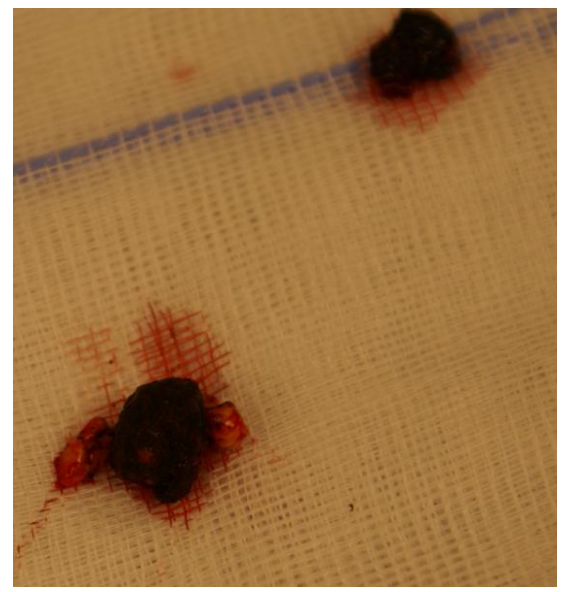

Figure 6. Gallstones found in the abcess.

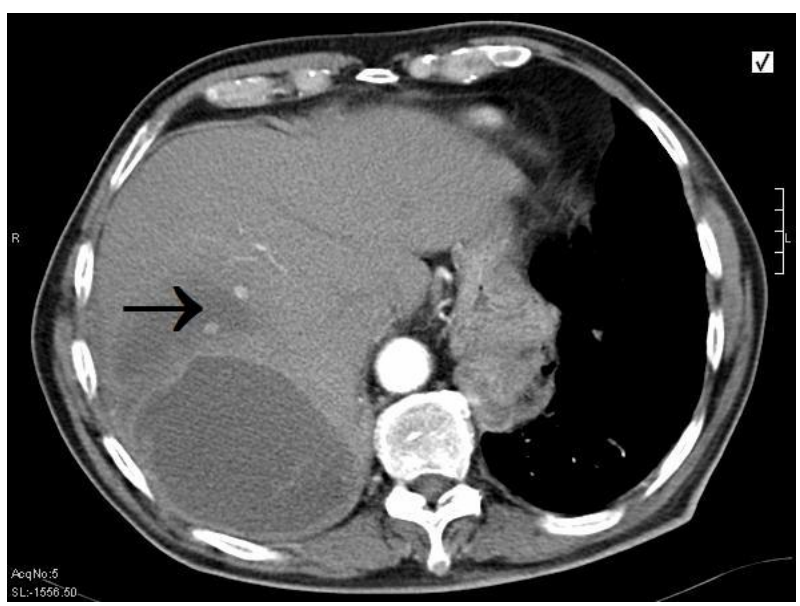

Figure 7. Careful examination of the CT scan performed at the referring clinic already showed the gallstones. 
The infiltration in the lower right lung resulted in a pleural empyema, which was surgically evacuated. Klebsiella species were found in both sputum and the liver abcess, but not in the empyema. Initially, cefuroxim was administered intravenously, and later replaced by amoxicillin/clavulanic acid when the patient's condition had improved. Antibiotic treatment was given for eleven weeks. The patient made a full recovery. The cholecystectomy report confirmed there had been spill of gallbladder contents at the time, although spillage of stones had not been reported.

\subsection{Patient D}

A 67-year old male was admitted to our hospital with recurring fever. Medical history showed cystic kidneys, with terminal renal insufficiency. Fifteen years earlier patient had received his second, still functional, renal transplant. Several episodes of urosepsis had occured in the past years. Further medical history included an appendectomy, atrial fibrillation and intermediate left ventricular function. Current medication included prednisolone and azathioprine.

Several episodes of fever and bacteriaemia with E Coli had occured in the previous weeks. It was concluded that the infection had originated in the still present cystic kidneys. Laparoscopic nefrectomy of the left cystic kidney was performed, followed by an episode of diverticulitis. CT scan at that time also showed a dilated biliary tract, without an obstructing moment. It was decided to delay nefrectomy of the right kidney for several months in order to let patient recuperate.

Two weeks later, patient presented again with abdominal dyscomfort, anorexia with $10 \mathrm{~kg}$ of weight loss and a fever. Abdominal ultrasound showed no signs of an abscess, nor any other focus of inflammation. Blood cultures remained negative. No antibiotics were administered, but the dosage of prednisolone was temporarily increased. Patient made a recovery, after which he was released.

Three weeks later, patient again presented with fever, anorexia, weight loss and abdominal dyscomfort, mostly in the lower right quadrant. Physical examination showed a low blood pressure $(80 / 50 \mathrm{mmHg}$, pulse 70 beats per minute under beta blockade). Laboratory tests showed moderately elevated CRP (Table 1). Abdominal ultrasound showed no signs of an abscess or inflammation. The left intrahepatical biliary duct system and the choledochal duct were dilated. An abdominal CT scan with iv contrast was performed, without any new findings. Because it was now believed the dilated biliary system might indicate an obstructive moment such as a pancreatic mass, even though there were no laboratory findings present indicative of cholestasis, an MRCP
(Figure 8) was performed. This showed multiple biliary stones in the pancreatic region, without any sign of a pancreatic mass. An ERCP was performed, which confirmed choledocholithiasis. After papillotomy, extraction of multiple stones was performed. During a second and third ERCP, performed because of persistent fever after the first intervention, no residual stones were found. Patient made a good recovery on amoxycillin/clavulanic acid administered for three weeks in total.

Whilst waiting for an elective cholecystectomy, patient developed an acute cholecystitis, for which cholecystectomy was performed. Patient has had several recurrences of choledocholithiasis afterwards.

\subsection{Patient E}

An 82-year old man presented at our Emergency unit with acute epigastric pain, nausea and vomiting. Medical history included COPD and a pulmonary embolism two years before presentation. Physical examination showed a septic patient, with an almost silent bowel, epigastric tenderness and muscular defence. Laboratory tests revealed iron deficiency anaemia, severe leukocytosis, slightly elevated CRP, as well as elevated serum and urine amylase. Liver tests were all in the normal range (Table 1). An abdominal ultrasound was performed, which showed aerobilia, a dilated choledochal duct and an enlarged pancreas. No biliary stones were visualized. Abdominal CT scan showed a pancreatitis and aerobilia (Figure 9). The gallbladder was located adjacent to the duodenum (Figure 10). A cholecystoduodenal fistula was suspected, and later confirmed by esophago-gastroscopy and ERCP. Several gallstones, as well as food, were removed from the distal choledochal duct after papillotomy was performed.

In the following days patient developed a full blown sepsis due to infected necrotic pancreatitis, for which drainage was achieved percutaneously under CT guidance. Gentamycin, cefuroxim and metronidazole were

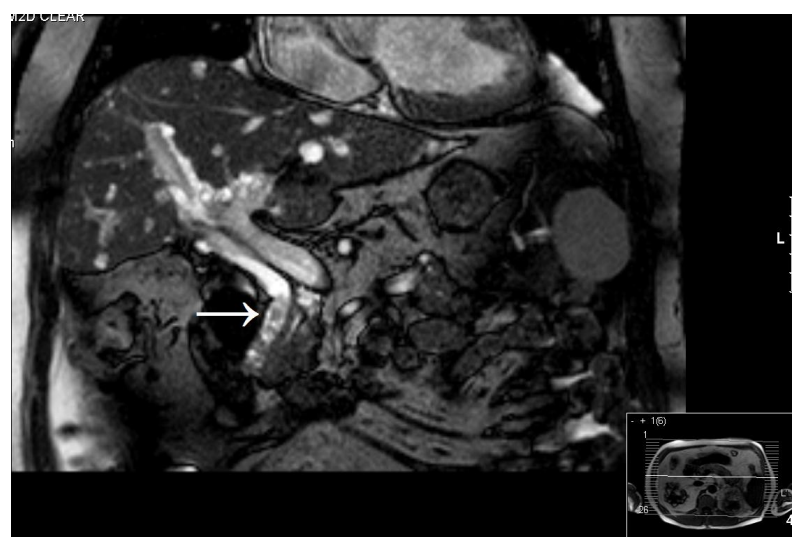

Figure 8. The MRCP shows biliary stones, as pointed out by the arrow. 


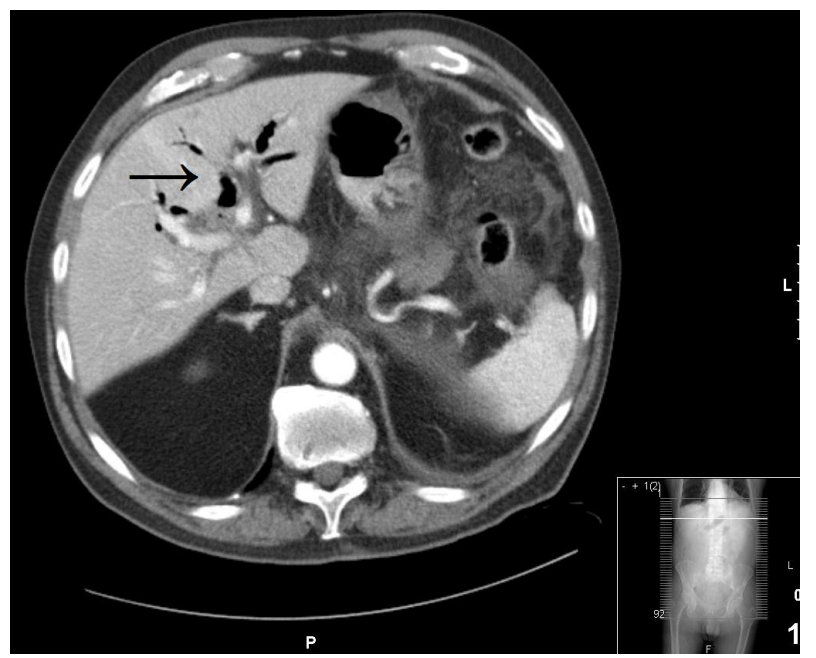

Figure 9. CT scan showing aerobilia.

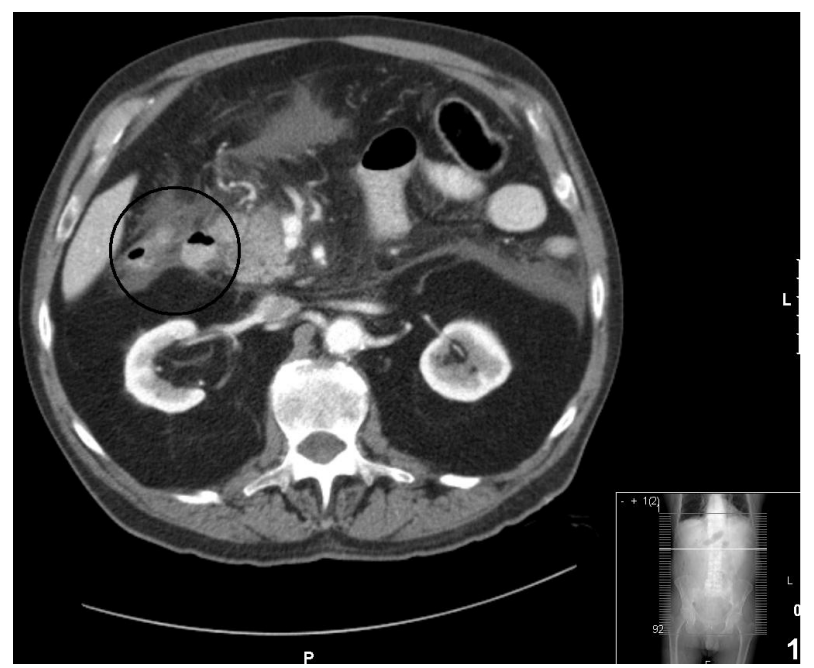

Figure 10. Same CT scan, showing the close relation between gallbladder and duodenum, respectively on the left and right side of the circle, within an inflamed region.

administered. Cultures of the drain fluids showed multiple enteric bacteriae. Blood cultures showed Streptococcus anginosus. Antibiotic treatment was then switched to tazobactam/piperacillin.

In the following weeks, the patient's condition fluctuated. Via endoscopic ultrasound an enteral drainage of the abscess was achieved several times. In spite of this, his condition eventually deteriorated, and the patient died.

\section{DISCUSSION}

With a prevalence of 5\% - 25\%, and in some groups even up to $75 \%$, gallstone disease is a common illness [1,3-5]. A wide variety of risk factors is currently known (Table 2), amongst which are obesity, female gender, increasing age, pregnancy and age. Despite their high
Table 2. Risk factors for biliary disease [1,3-5].

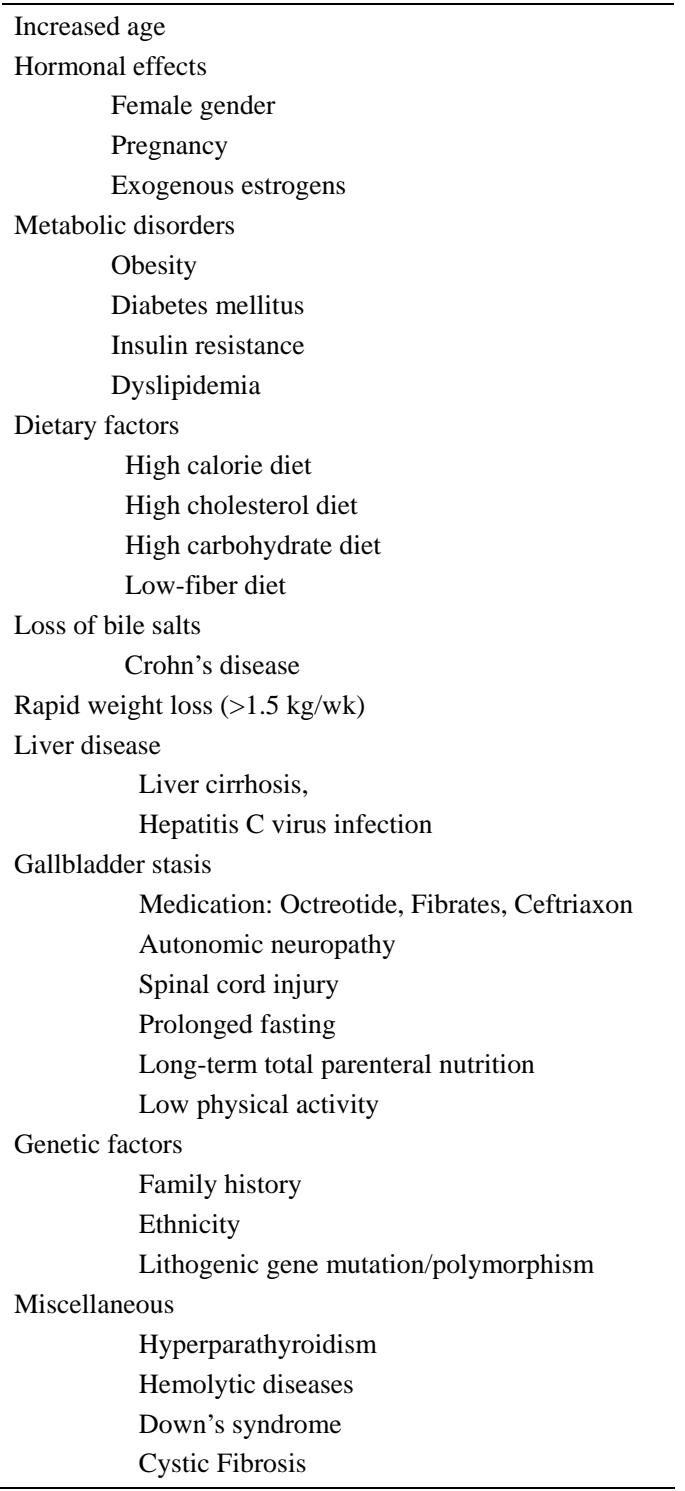

prevalence, only $10 \%$ - 30\% of gallstones are symptomatic [1,5].

Cholesterol stones account for $80 \%$ - $90 \%$ of all stones in Western countries [3]. The remainder are pigment stones.

If symptomatic, the most common presentation is that of biliary colics or epigastric discomfort [1,3]. More complications of gallstone disease are displayed in Table 3. Gallstone disease usually presents itself by either its complications, or by accidental discovery during diagnostic investigations such as an abdominal ultrasound [1].

In this article we describe several uncommon presentations of gallstone disease. Both patients $\mathrm{A}$ and $\mathrm{E}$ presented with a cholecystoduodenal fistula due to stone erosion through the gallbladder wall. In patient A this led to a gallstone ileus. Beyond causing iron deficiency 
Table 3. Complications of gallstone disease [3].

\begin{tabular}{lc}
\hline Common complications: & Anual Risk (\%) \\
\hline biliary colic & 1 \\
acute cholecystitis & 0.3 \\
symptomatic choledocholithiasis & 0.2 \\
cholangitis & \\
obstructed neck of the gallbladder & \\
mucocele & \\
extra-hepatic cholestasis & \\
pancreatitis & \\
\hline Rare complications: & \\
\hline gallbladder carcinoma & \\
liver abscess & \\
gallstone ileus & \\
gallbladder perforation/cholecysto-duodenal fistula & \\
\hline
\end{tabular}

anaemia due to blood loss, gall stone disease also led to an eventually fatal pancreatitis in patient $\mathrm{E}$.

Patient B showed a liver abcess, most likely as a complication of intermittent cholestasis and cholangitis due to gallstones. Patient $\mathrm{C}$ displayed a hepatic abscess due to gallstone spill during laparoscopic cholecystectomy several years earlier. Patient D had intermittent bacteraemias and atypical abdominal complaints under immunosuppressive therapy because of a renal transplant. Despite nephrectomy the bacteraemia relapsed. Eventually cholelithiasis was revealed, for which stone removal and cholecystectomy were performed. Despite this, patient suffered recurrent gall stone disease.

Below we will elaborate on these presentations.

Gallstone ileus results from recurrent attacks of cholecystitis with erosion of a gallstone through a fistula through the adjacent duodenal wall into small intestine $[4,6,7]$. Normally the fistula will be formed between a gangrenous gallbladder and the duodenum (cholecystoduodenal fistula). Occasionally the fistula forms between the gallbladder and other parts of the gastrointestinal tract [6,7]. Patient E presented which aerobilia on initial imaging, which (apart from secondary to operation or papillotomy) can only be caused by such a fistula [7].

Gallstone ileus accounts for $1 \%$ - 3\% of mechanical small bowel obstruction [6] and causes up to 25\% of cases of non-strangulated small bowel obstruction in patients over 65 years of age [6,7]. Mean age at presentation is 65 - 75 years and it is 3 times more common in women than men $[6,7]$. Clinical symptoms may be ileus, intermittent obstipation or diarrhoea, melaena, acute upper gastro-intestinal bleeding and aerobilia [4,6,7]. More than one-third of patients present with no history of biliary symptoms and $40 \%$ - 50\% with a history of symptomatic cholelithiasis [6].

Overall mortality rate varies between $12 \%$ - 33\% [4,7]. This may be attributed to delayed diagnosis, elderly patients, and coexisting concomitant medical disease. An accurate preoperative diagnosis is made in $20 \%-73 \%$ of patients [6,7]. CT scanning appears most sensitive to reveal gall stone ileus [6]. Although other treatment modalities, such as ERCP, are employed, the treatment of choice is surgery [6,7]. Enterolithotomy alone has been shown to have a lower mortality rate on both the short and long term than enterolithotomy combined with fistula repair [6]. Re-operation rate for secondary fistula repair or recurrence of ileus were low (10\% and 5\% - 9\% respectively) [6].

Liver abscess is the most common type of visceral abcess, accounting for $48 \%$ of visceral abscesses, and $13 \%$ of all intra-abdominal abscesses [8]. The incidence of liver abscess has been estimated at 2.3 per 100.000 citizens [9]. It is more common among men (3.3 vs 1.3 per 100.000) [9]. Biliary tract obstruction, due to either malignancy or gallstones, accounts for $40 \%$ - $60 \%$ of pyogenic liver abscesses [8-10]. Most abscesses are polymicrobial, mostly due to facultative or anaerobic species from the intestinal tract $[8,9]$.

Presentation of liver abscess usually includes fever and abdominal pain, as well as nausea, vomiting, malaise, weight loss and anorexia [9]. Depending on the size and location of the abcess there may be hepatomegaly or jaundice. Imaging should be performed by 4 phase CT scan or ultrasound [8].

The best treatment for hepatic abscess is still uncertain $[8,10,11]$. Current convention is that treatment should include both drainage, either operatively or radiologically, and long-term antibiotics. The main guide in this is the clinical condition of the patient. Despite optimal treatment, mortality of liver abscess remains high. Although usually around $6 \%$, it can be as high as $53 \%$ in elderly patients [12,13].

In the case of patient $B$, it is remarkable that during her first hospital stay, neither abdominal ultrasound, nor CT scan, showed signs of gallstone disease, even though sensitivity of these combined diagnostic tools exceeds $84 \%$ and specificity of ultrasound alone is $99 \%[2,5]$.

Laparoscopic cholecystectomy is the treatment of choice for both acute cholecystitis and other presentations of symptomatic gallstone disease $[4,14,15]$. Reduced cost, decreased hospital stay and increased patient satisfaction are amongst the advantages of this technique [15]. On the other hand laparoscopic cholecystectomy has a higher rate of several complications, such as bile duct injury. Some complications are hardly ever seen in open cholecystectomy, such as visceral laceration or gallstone spillage into the abdominal cavity [6]. The incidence of gall bladder perforation during laparoscopic surgery has been reported to be $20 \%$ - 40\% [6,16,17]. Gallstone spill occurs in 7\% - 16\% of all laparoscopically performed cholecystectomies [16,17]. 
Not much is known about the natural history of spilled gallstones. It is estimated that about $2 \%$ of all spilled stones give rise to clinical problems [16]. This can occur days to years after the initial surgery [6]. The usual presentation is in the form of an abscess, either intraabdominally or in the operation tract [6]. Pseudocysts have also been reported [18]. Predisposing factors for developing complications after stone spill are reported to be older age, male sex, acute cholecystitis at the time of operation, spillage of pigment stones, number of stones $(>15)$ or size of the stone (diameter $>1.5 \mathrm{~cm}$ ), and perihepatic localization of lost stones [6,17]. If possible, spill of stones should of course be avoided, and retrieving spilled stones should be considered [6,17].

The age of the patients presented in this article exceeds 65 years in all cases. All of them showed serious morbidity due to gallstone disease. In general, these cases of relatively uncommon, but otherwise well-known complications of gallstone disease, demonstrate that in elderly patients with atypical abdominal complaints gallstone disease should always be considered.

Most of the uncommon complications of gallstone disease are more common in the elderly, as is gallstone disease in general. Fifteen percent of men and $24 \%$ of women have gallstones at the age of 70 . At 90 years, those numbers rise to $24 \%$ and $35 \%$ respectively [15].

Typical symptoms such as colics, local peritonitis and fever are often absent in these patients. This also counts for immunocompromised patients (HIV patients, use of immunosuppressives or corticosteroids) and patients on hemodialysis, as case D clearly demonstrates.

Although surgeons might be hesitant to operate on elderly patients, we would advocate at least serious consideration on a case-to-case basis. Not only is the average patient getting older, but operating on the elderly has been proven to be safe $[14,15,19,20,21]$. The main reason for higher morbidity among the elderly is comorbidity $[14,19,20]$. Careful selection and preoperative optimalization can minimize any risks. Some authors even state this judicous selection and preparation would only be required in those over 80 years [14]. Furthermore, Riall showed that in those elderly patients in whom cholecystectomy is not performed after a first complication of gallstone disease, readmission rate is $38 \%$ in the following two years, most of whom require cholecystectomy. In those operated the readmission rate is only $4 \%$ [15].

As mentioned, comorbidity is one of the main reasons not to operate on elderly patients. Although comorbidity predicts 2-year mortality, lack of definitive therapy in complicated gallstone disease is an independent predictor of mortality, suggesting increased mortality rate in conservative treatment [15].

\section{CONCLUSION}

This case series describes several uncommon, but generally known complications of gallstone disease. It is demonstrated that gallstone disease should always be considered in case of elderly or immunocompromised patients with atypical (abdominal) symptoms or radiologic findings. It also shows that in those patients who present with symptomatic gallstone disease, cholecystectomy should not be dismissed easily.

\section{REFERENCES}

[1] Yoo, E.H. and Lee, S.Y. (2009) The prevalence and risk factors for gallstone disease. Clinical Chemistry and Laboratory Medicine, 47, 795-807. doi:10.1515/CCLM.2009.194

[2] Zakko, S. and Ramsby, G. (1990) Role of computed tomography in assessing gallstones. Radiology Report, 2, 426.

[3] Attasaranya, S., Fogel, E.L. and Lehman, G.A. (2008) Choledocholithiasis, ascending cholangitis, and gallstone pancreatitis. Medical Clinics of North America, 92, 925960. doi:10.1016/j.mcna.2008.03.001

[4] Sanders, G. and Kingsnorth, A.N. (2007) Gallstones. British Medical Journal, 335, 295-299. doi:10.1136/bmj.39267.452257.AD

[5] Marschall, H.U. and Einarsson, C. (2007) Gallstone disease. Journal of Internal Medicine, 261, 529-542. doi:10.1111/j.1365-2796.2007.01783.x

[6] Zaliekas, J. and Munson, J.L. (2008) Complications of gallstones: The Mirizzi syndrome, gallstone ileus, gallstone pancreatitis, complications of "lost" stones. Surgical Clinics of North America, 88, 1345-1368. doi:10.1016/j.suc.2008.07.011

[7] Abou-Saif, A. and Al-Kawas, F.H. (2002) Complications of gallstone disease: Mirizzi syndrome, cholecystocholedochal fistula, and gallstone ileus. The American Journal of Gastroenterology, 97, 249-254. doi:10.1111/j.1572-0241.2002.05451.X

[8] Mezhir, J.J., Fong, Y. and Jacks, L.M. (2010) Current management of pyoegnic liver abscess: Surgery is now second-line treatment. Journal of the American College of Surgeons, 210, 975-983. doi:10.1016/j.jamcollsurg.2010.03.004

[9] Huang, C.-J., Pitt, H.A., Lipsett, P.A., et al. (1996) Pyogenic hepatic abscess: Changing trends over 42 years. Annals of Surgery, 223, 600-607. doi:10.1097/00000658-199605000-00016

[10] Lam, Y.-H., Wong, S. K.-H., Lee, D.W.-H, et al. (1999) ERCP and pyogenic liver abscess. Gastrointest Endosc, 50, 340-344. doi:10.1053/ge.1999.v50.98065

[11] Ch-Yu, S., Hg-Lo, R., Kan, P.S. and Metreweli, C. (1997) Pyogenic liver abscess: Treatment with needle aspiration. Clinical Radiology, 52, 912-916. doi:10.1016/S0009-9260(97)80223-1

[12] Lee, K.T., Wong, S.R. and Sheen, P.C. (2001) Pyogenic 
liver abscess: An audit of 10 years' experience and analysis of risk factors. Digestive Surgery, 18, 459-465. doi:10.1159/000050194

[13] Sridharan, G.V., Wilkinson, S.P. and Primrose, W.R. (1990) Pyogenic liver abscess in the elderly. Age Ageing, 19, 199-203. doi:10.1093/ageing/19.3.199

[14] Yetkin, G. and Uludag, M. (2009) Laparoscopic cholecystectomy in elderly patients. Journal of the Society of Laparoendoscopic Surgeons, 13, 587-591. doi:10.4293/108680809X1258998404604

[15] Riall, T.S. and Zhang, D. (2010) Failure to perform cholecystectomy for acute cholecystitis in elderly patients is associated with increased morbidity, mortality, and cost. Journal of the American College of Surgeons, 210, 668677.

[16] Woodfield, J.C., Rodgers, M. and Windsor, J.A. (2004) Peritoneal gallstones following laparoscopic cholecystectomy: Incidence, complications, and management. Surgical Endoscopy, 18, 1200-1207. doi:10.1007/s00464-003-8260-4

[17] Brockmann, J.G., Kocher, T., Senninger, N.J. and Schür- mann, G.M. (2002) Complications due to gallstones lost during laparoscopic cholecystectomy. Surgical Endoscopy, 16, 1226-1232. doi:10.1007/s00464-001-9173-8

[18] Arishi, A.R., Rabie, M.E., Khan, M.S., Sumaili, H., Shaabi, H., Michael, N.T. and Shekhawat, B.S. (2008) Spilled gallstones: The source of an enigma. Journal of the Society of Laparoendoscopic Surgeons, 12, 321-325.

[19] Osman, Y., Ebru, M., Umit, T., Volkan, O., Melih, K., Belma, K., Betul, B., Omer, C. and Atilla, S. (2008) Laparoscopic cholecystectomy in elderly patients. Bratislava Medical Journal, 109, 313-316.

[20] Mayol, J., Martinez-Sarmiento, J., Tamayo, F.J. and Fernández-Represa, J.A. (1997) Complications of laparoscopic cholecystectomy in the ageing patient. Age Ageing, 26, 77-81. doi:10.1093/ageing/26.2.77

[21] Sánchez-Beorlegui, J., Soriano, P., Monsalve, E., Moreno, N., Cabezali, R. and Navarro, A. (2009) Laparoscopic cholecystectomy in octogenarian patients. A comparative study between two geriatric population groups. Cirugía Española, 85, 246-251. 